\title{
The feature-positive effect in pigeons: Conditionality, overall predictiveness, and type of feature
}

\author{
ELIOT HEARST \\ Indiana University, Bloomington, Indiana
}

\begin{abstract}
Two experiments examined variations of standard visual discrimination arrangements in which pigeons typically perform much better when a distinguishing feature appears on reinforced (featurepositive) than on nonreinforced (feature-negative) trials. In Experiment 1 the feature was continuously present on the response key during intertrial intervals as well as during the appropriate type of discrimination trial. Although retarded in its appearance, a strong feature-positive superiority did occur. In Experiment 2 the feature and common cues were reversed in function from those in conventional procedures; a small white square was common to all trials, and the distinctive element involved illumination of the key with a green color. Nevertheless, a significant feature-positive superiority was found, although excellent performance was achieved rapidly for both groups. The results suggest that the conditionality versus nonconditionality of the two discriminations and the feature's overall positive predictiveness of food are not crucial in producing superior discriminative performance in the feature-positive case.
\end{abstract}

When a single distinguishing feature (e.g., a small white square on a response key) is present on positive trials and absent on negative trials, pigeons show much better discriminative performance than under the reverse conditions. This superiority of the feature-positive (FP) over the feature-negative (FN) arrangement has considerable interspecies generality and occurs in a wide variety of behavioral settings (for reviews, see Hearst, 1978, 1984; Hearst \& Jenkins, 1974; Jenkins \& Sainsbury, 1969, 1970). However, no proposed explanation or theory satisfactorily encompasses all the data.

Jenkins and his coworkers (Bitgood, Segrave, \& Jenkins, 1976; Jenkins \& Sainsbury, 1970) pointed out one aspect of the standard procedure that could help account for many instances of the FP superiority: The FP subjects have only to associate the mere presence of the feature with reinforcement in order to perform perfectly on the discrimination (i.e., they can follow the simple rule "respond if the feature is present"). In contrast, FN subjects must learn to withhold responding to cues common to all trials (say, illumination of a colored key) depending on the presence of the feature (i.e., their rule would be "given a trial, respond if the feature is absent"). In other words, the FN arrangement entails a conditional discrimination and the FP does not; therefore performance on the latter should be superior.

Hearst and Jenkins (1974) suggested that sign-tracking might often play a crucial role in producing the FP-FN

This research was supported by National Institute of Mental Health Grant MH 19300. I thank Dexter Gormley, Diana Lee, Michael Mondloch, and Robert Sampson for assistance and advice. Correspondence concerning this article should be sent to Eliot Hearst, Department of Psychology, Indiana University, Bloomington, IN 47405. differences. Organisms have a natural tendency to approach and contact positive signs of appetitive reinforcement, and, if they direct their behavior toward the best positive predictor of food in a situation, FP performance should be considerably better than FN performance. On standard procedures with intertrial intervals (ITIs), the best positive predictor in the FP arrangement is the feature itself (present only on positive trials), whereas the best predictor in the FN arrangement consists of the common trial cues (present on both positive and negative trials). In support of this analysis, pigeons on FP procedures come to peck directly at the feature, whereas pigeons on the FN procedure peck at other portions of the display and almost never at the feature.

My first experiment examined whether such differences in conditionality and overall predictiveness were necessary to produce an FP superiority. On our standard procedure for studying the phenomenon in keypecking pigeons (see Hearst, 1987), trials with a white square on a lighted green background are interspersed with presentations of the lighted green background alone. The key is dark during ITIs. I wondered what would happen if the square were also presented continuously during ITIs. Both the FP and FN discriminations would then become conditional (given a trial, food would follow if the square was present or absent, respectively). Furthermore, within this arrangement the feature would be nonpredictive of food because of its constant presence. If the FP superiority were still obtained under these conditions, simple explanations in terms of conditionality or overall predictiveness of the feature would be untenable.

The second experiment had a different focus from the first. In most studies of the FP superiority, the feature has been a small visual element, less salient than the com- 
mon cues appearing on all trials. However, suppose the reverse kind of arrangement held, with the presence of colored areas serving as the feature and the square as the common element. Simultaneous appearance of the square and color would constitute a feature trial, whereas appearance of the square alone would constitute a nonfeature trial. Would such a reversal of the feature and common cues lead to a disappearance of the FP effect, and possibly even an advantage for the FN subjects? Furthermore, data from such research could help in defining what a "feature" is, along lines similar to Treisman and Souther's (1985) use of the so-called pop-out effect as one diagnostic for isolating and characterizing primitive features.

\section{EXPERIMENT 1}

\section{Method}

Subjects. Sixteen experimentally naive adult female White Carneaux pigeons, maintained at $75 \%$ of their free feeding weights, served as subjects.

Apparatus. Two standard pigeon chambers were employed, whose details were described more fully by Hearst (1987). The response key was centered $14 \mathrm{~cm}$ above the grain aperture in both chambers. After the first few magazine training trials, all grain presentations lasted $3 \mathrm{sec}$. An in-line projector permitted illumination of the key with three types of visual displays: (1) a homogeneous green field, (2) the green field with a small $(0.2-\mathrm{cm})$ white square at its center, or (3) the small white square against an unlit background. A houselight remained on continuously throughout sessions.

Procedure. All birds spent two sessions learning to eat whenever the food tray was operated, according to our standard magazine training procedure (see Hearst, 1987). The key was dark during this training. Then the subjects were randomly assigned to either the FP $(n=8)$ or the FN $(n=8)$ condition for discrimination training, which began on Day 3. Each such session consisted of 406 -sec stimulus periods, separated by ITIs averaging $60 \mathrm{sec}$ (range: $15-105 \mathrm{sec}$ ). Half the stimulus periods involved illumination of the key with green light only (nonfeature trials) and half with the green + square display (feature trials). During ITIs the small white square appeared continuously at the center of the otherwise unlit key. The key was completely dark only during the brief times when grain was available in the magazine.

An autoshaping procedure was in force, and all grain deliveries were response independent. For FP subjects, food immediately followed all feature trials $(\mathrm{CS}+)$, and nothing happened after nonfeature trials (CS-). For FN subjects, food immediately followed all nonfeature trials (CS+), and nothing followed feature trials (CS-). Sequences of the positive and negative stimuli were irregular, except for the constraint that neither type of stimulus could occur more than three consecutive times. After 10 days of discrimination training, complete extinction was introduced for 5 days; food did not follow either the previous $\mathrm{CS}+$ or $\mathrm{CS}-$. The extinction phase was included to check the generality of our prior finding that removal of all reinforcement usually leads to a revelation or improvement of discriminative control (Hearst, 1987).

\section{Results and Discussion}

Figure 1 displays discrimination ratios over the two phases of the experiment. These ratios were calculated by dividing the number of keypecks on positive trials by the total number of keypecks on both positive and negative trials: Thus a ratio of .50 indicates no difference in responding between the two types of trials, and a ratio of 1.00 represents perfect performance. Neither the FP nor the FN subjects showed substantial evidence of dis-

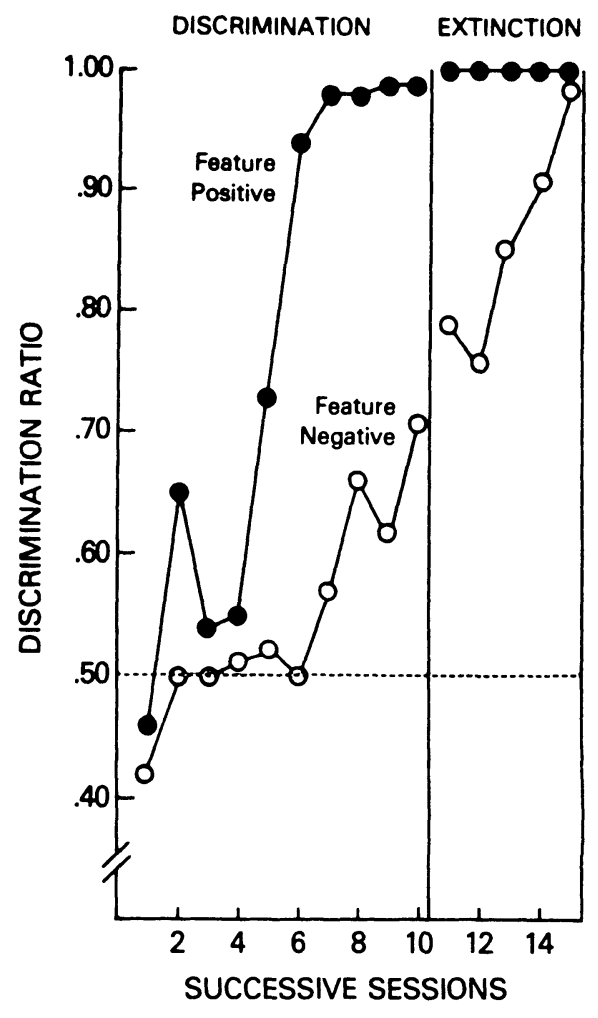

Figure 1. Median discrimination ratios for feature-positive and feature-negative pigeons given discrimination training for 10 sessions and then extinguished to both the positive and the negative stimulus for 5 sessions. The feature (a small white square) was continuously present during intertrial intervals. (Experiment 1)

crimination learning over the first 4 training sessions, but the FP subjects rapidly improved and performed at a very high level during Sessions 6-10. On the other hand, the FN subjects did not attain excellent performance and were inferior to the FP subjects throughout discrimination training.

Statistical analyses of FP-FN differences were conducted to compare discrimination ratios over Sessions 2-10 (the first session was not included because 4 birds in the FP group and 2 birds in the FN group did not peck the key at all during that session, and consequently ratios could not be calculated for them). The main effects of groups and sessions were highly significant $[F(1,14)=$ $53.70, p<.001$, and $F(8,112)=19.73, p<.001$, respectively], as was the interaction between them $[F(8,112)=5.09, p<.001]$. Thus a clear featurepositive superiority was obtained in the present experiment, although FP birds showed considerably lower levels of performance during Sessions 2-5 than on standard procedures without presentation of the feature during ITIs (see Figure 1 in Hearst, 1987). The FN birds improved more over Sessions 7-10 than is normally observed in our laboratory (see especially Experiments 1,3 , and 4 in Hearst, 1987), but no FN bird attained a discrimination ratio greater than .86 during Session 10 , whereas 7 of the 8 FP birds did better than .96 on that day. 
Thus the continuous presence of the square during ITIs retarded the appearance of a large FP superiority, but did not remove it. The conditional nature of the FP arrangement in this experiment suggested that FP birds may first have to extinguish responding to the square during ITIs before the FP discrimination clearly appears; in other words, subjects must learn to differentiate between squares on trials and squares during ITIs. An ANOVA on the daily number of ITI responses for Sessions 2-10 revealed a significant decline over those days in the FP group $[F(8,112)$ $=4.07, p<.001]$, but not in the FN group $[F(8,112)$ $=1.40]$. However, the absolute daily number of ITI responses differed significantly between the FP and FN groups during Session 3 only $[F(1,126)=7.28$, $p<.01]$.

Figure 1 also supplies another example of the extinction-revelation effect reported by Hearst (1987). The FN subjects showed much better discrimination ratios during the extinction phase (Sessions 11-15) than during the last 5 days of initial training (Sessions 6-10) $[t(7)=3.39$, $p<.02]$. The $5 \mathrm{FN}$ birds that exhibited the lowest median ratios during Sessions 6-10 (ranging from .38 to .61) displayed median ratios ranging from .72 to .97 during the extinction phase. This outcome supports the conclusion of our earlier report, namely that failures to display FN acquisition reflect a deficiency in control of performance rather than a lack of learning.

\section{EXPERIMENT 2}

\section{Method}

Subjects and Apparatus. Twelve experimentally naive subjects, of the same type and maintained in the same way as in Experiment 1, were used. The test chambers and other apparatus details were identical to those in Experiment 1.

Procedure. After standard magazine training, birds were randomly assigned to either an FP $(n=6)$ or an FN $(n=6)$ condition for discrimination training, which began on Day 3 . All aspects of the procedure matched those in Experiment 1 except that the key was dark during ITIs and that the 40 stimulus periods included 20 presentations of the white square on the green background and 20 presentations of the white square alone. The former (feature) trial type served as the CS+ for the FP birds and the CS- for the FN birds; the latter (nonfeature) trial type served as the CS- for FP birds and the CS+ for FN birds. Subjects remained on their respective discriminations for 10 days and were then placed on extinction for 5 days. During the last 3 days of the discrimination phase, the experimenter periodically observed each bird and recorded the target of its pecking responses-green areas of the key or the white square.

\section{Results and Discussion}

Excellent FP and FN discriminations appeared rapidly under this reversal of the feature-common assignments of previous studies. Nevertheless, an FP superiority was still obtained when FP and FN ratios were analyzed over Sessions 2-10. The interaction between groups and sessions was statistically significant $[F(8,80)=3.58$, $p<.01]$, and the main effect of groups reached a $p$ level of .06 $[F(1,10)=4.76]$. The main effect of sessions was highly significant $[F(8,80)=4.77, p<.001]$. Figure 2 shows that by the fourth training day, discrimination ra-

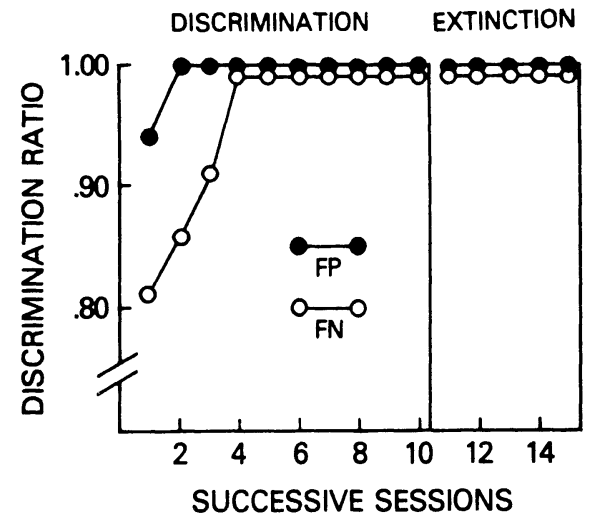

Figure 2. Median discrimination ratios for feature-positive (FP) and feature-negative (FN) pigeons given discrimination training for 10 sessions and then extinguished to both the positive and the negative stimulus for 5 sessions. The feature was a green field on the response key and the common cue was a small white square, which is a reversal of the standard arrangement. (Experiment 2)

tios were excellent in both groups. From Sessions 4-10 all 12 individual subjects yielded a daily ratio of 1.00 . Of course, performance prior to Session 4 accounted for the finding of a significant FP superiority.

Periodic observation of every bird during Sessions 8-10 revealed consistent but different targets of directed pecking at CS+ in the FP and FN groups. The FP birds pecked somewhere on the green part of the key, usually at the top, bottom, or another place on the key's perimeter, whereas the FN birds pecked at or very close to the white square in the center of the key. Therefore, the pecking locations with the green stimulus as the feature were the opposite of those observed for FP and FN birds in prior work with the square as the feature. However, a reliable observation in all these arrangements was that birds peck at the best positive predictor of food in the situation.

Performance was so good during Sessions 4-10 that a ceiling effect prevented any possible detection of extinction-induced discriminative improvements during Sessions 11-15.

In corinparison with the standard procedure, use of the green stimulus as the feature greatly improved FP and Fiv acquisition performance but did not totally remove the FP advantage. This outcome parallels results (see Hearst, 1978; Morris, 1977) that indicate that arrangements with highly salient features typically yield a relatively weak or nonexistent FP superiority.

\section{GENERAL DISCUSSION}

Although the magnitude of the FP superiority was influenced by the manipulations in these two experiments, the general phenomenon was still obtained in both studies. This outcome occurred despite some good reasons for believing it might disappear if the feature were continuously present during ITIs or if it were distinctly more salient than common trial cues. The relative conditionality of the standard FP versus FN discriminations proved not to be a crucial factor, and elimination of the predictiveness of the feature's presence with respect to food delivery failed to remove the FP superiority (Experiment 1). It seems likely that, 
after some strong initial generalization between the stimulus conditions during trials and ITIs, subjects soon come to discriminate between them; and then differences in FP versus FN performance follow the more or less typical pattern. Arrangements in which ITIs are completely removed from the procedure-forcing both the FP and FN discriminations to be based solely on presence versus absence of a feature-ought to enable a deeper analysis of the relevant possibilities, especially with respect to the role of mere addition versus deletion as signals. Wolff (1983) examined certain discriminations of this kind, and the same theme guides some current research in our laboratory.

The improvement in FN discrimination performance that occurred during extinction in Experiment 1 replicates the results of Hearst (1987) and supports our prior conclusion that the FP superiority in standard pigeon experiments reflects a deficiency in control of $\mathrm{FN}$ performance rather than a lack of learning.

\section{REFERENCES}

Bitgood, S. C., Segrave, K., \& Jenkins, H. M. (1976). Verbal feedback and the feature-positive effect in children. Journal of Experimental Child Psychology, 21, 249-255.

HeARST, E. (1978). Stimulus relationships and feature selection in learning and behavior. In S. Hulse, H. Fowler, \& W. K. Honig (Eds.), Cognitive processes in animal behavior (pp. 51-88). Hillsdale, NJ: Erlbaum.

HearST, E. (1984). Absence as information: Some implications for learning, performance, and representational processes. In H. L. Roitblat,
T. G. Bever, \& H. S. Terrace (Eds.), Animal cognition (pp. 311332). Hillsdale, NJ: Erlbaum.

HEARST, E. (1987). Extinction reveals stimulus control: Latent learning of feature-negative discriminations in pigeons. Journal of $E x$ perimental Psychology: Animal Behavior Processes, 13, 52-64.

HEARST, E., \& JENkINS, H. M. (1974). Sign-tracking: The stimulusreinforcer relation and directed action. Austin, TX: The Psychonomic Society.

JENKINS, H. M., \& SAINSBURY, R. S. (1969). The development of stimulus control through differential reinforcement. In N. J. Mackintosh \& W. K. Honig (Eds.), Fundamental issues in associative learning (pp. 123-161). Halifax, NS, Canada: Dalhousie University Press.

Jenkins, H. M., \& SAINSBURY, R. S. (1970). Discrimination learning with the distinctive feature on positive or negative trials. In D. Mostofsky (Ed.), Attention: Contemporary theory and analysis (pp. 239-273). New York: Appleton-Century-Crofts.

MoRRIS, R. C. (1977). Spatial variables and the feature-positive effect. Learning \& Motivation, 8, 194-212.

Treisman, A., \& Souther, J. (1985). Search asymmetry: A diagnostic for preattentive processing of separable features. Journal of $E x-$ perimental Psychology: General, 114, 285-310.

WolfF, W. T. (1983). Discrimination learning based on the presence and absence of predictive stimuli: An analysis of the feature positive effect. Unpublished doctoral dissertation, Indiana University.

(Manuscript received for publication May 30, 1987.) 